\title{
Measurements and Analytics of Wide-Area File Transfers Over Dedicated Connections
}

\author{
Nageswara S. V. Rao \\ Oak Ridge National Laboratory \\ Oak Ridge, TN, USA \\ raons@ornl.gov \\ Zhengchun Liu \\ Argonne National Laboratory \\ Argonne, IL, USA \\ zhengchun.liu@anl.gov
}

\author{
Qiang Liu \\ Oak Ridge National Laboratory \\ Oak Ridge, TN, USA \\ liuq1@ornl.gov \\ Raj Kettimuthu \\ Argonne National Laboratory \\ Argonne, IL, USA \\ kettimut@anl.gov
}

\author{
Satyabrata Sen \\ Oak Ridge National Laboratory \\ Oak Ridge, TN, USA \\ sens@ornl.gov \\ Ian Foster \\ Argonne National Laboratory \\ Argonne, IL, USA \\ foster@anl.gov
}

\begin{abstract}
Distributed scientific and big-data computations are becoming increasingly dependent on access to remote files. Wide-area file transfers are supported by two basic schemes: (i) application-level tools, such as GridFTP, that provide transport services between file systems housed at geographically separated sites, and (ii) file systems mounted over wide-area networks, using mechanisms such as LNet routers that make them transparently available. In both cases, the file transfer performance critically depends on the configuration consisting of host, file, IO, and disk subsystems, which are complex by themselves, as well as on their complex compositions implemented using buffers and IO-network data transitions. We present extensive file transfer rate measurements collected over dedicated 10 Gbps connections with 0-366 ms round-trip times, using GridFTP and XDD file transfer tools, and Lustre file system extended over wide-area networks using LNet routers. Our test configurations are composed of: three types of host systems; XFS, Lustre, and ext3 file systems; and Ethernet and SONET wide-area connections. We present analytics based on the convexity-concavity of throughput profiles which provide insights into throughput and its superior or inferior trend compared to linear interpolations. We propose the utilization-concavity coefficient, a scalar metric that characterizes the overall performance of any file transfer method consisting of specific configuration and scheme. Our results enable performance optimizations by highlighting the significant roles of (i) buffer sizes and parallelism in GridFTP and XDD, and (ii) buffer utilization and credit mechanism in LNet routers.
\end{abstract}

\section{CCS CONCEPTS}

- Networks $\rightarrow$ Network performance evaluation; Network measurement;

Keywords and phrases: Lustre file system, wide-area networks, throughput profile, network measurements.

ACM acknowledges that this contribution was authored or co-authored by an employee contractor, or affiliate of the United States government. As such, the United States government retains a nonexclusive, royalty-free right to publish or reproduce this article, or to allow others to do so, for government purposes only.

ICDCN '19, fanuary 4-7, 2019, Bangalore, India

(C) 2019 Association for Computing Machinery.

ACM ISBN 978-1-4503-6094-4/19/01...\$15.00

https://doi.org/10.1145/3288599.3288641

\section{INTRODUCTION}

Big data and science applications are becoming increasingly distributed, and they often require coordinated computations at geographically distributed sites that require access to files over WideArea Networks (WANs). Typically, file systems are installed at local sites, and wide-area file transfers are carried out by tools, such as Globus [4], Aspera [3], XDD [23], UDT [5], MDTM [10], and others. Another somewhat less frequently used scheme is based on enhancing the infrastructure to mount file systems over WAN $[6,11]$, thereby making them transparently available at remote sites. In particular, the Lustre file system is typically implemented over an InfiniBand (IB) site network, and is extended to Ethernet WAN using LNet routers [14]. File transfer throughputs achieved in these scenarios vary significantly based on two factors: (i) configuration that consists of file and storage system, transfer hosts and network connections, and (ii) file transfer scheme, such as GridFTP or LNet router. We collectively refer to both as the file transfer method. In this paper, we consider file transfers that utilize the Transmission Control Protocol (TCP) for underlying data transport over dedicated connections, such as those provisioned by ESnet's OSCARS [12] and Google's Software Defined Network (SDN) [7].

The performance of a file transfer method is characterized by its throughput profile $\hat{\Theta}_{E}(\tau)$ that specifies throughput achieved over a connection with Round-Trip Time (RTT) $\tau$. A throughput profile critically depends on the configuration, and equally importantly, on compositions of file systems and network connections at the sites, which involve matching file IO with network transport through buffer management. For example, over dedicated 10GigE connections with $\tau \in[0,366] \mathrm{ms}, \hat{\Theta}_{E}(\tau)$ of XDD for the XFS file system mounted on Solid State Device (SSD) storage is shown in Fig. 1(a), and that of Lustre file system extended using LNet routers is shown in Fig. 1(b). They represent two contrasting profiles: they differ evidently in peak throughput values, namely, 10 and $4.5 \mathrm{Gbps}$; additionally, the shape of the former is concave in contrast to the convex shape of the latter. The peak of a throughput profile is a direct indicator of the performance, and furthermore, its shape is subtler but also an important indicator, particularly, in projecting measurements to obtain throughput estimates $[9,15]$. Consider projecting $\hat{\Theta}_{E}(\tau)$ based on throughput measurements collected at RTTs $\tau_{1}$ and $\tau_{2}$, for $\tau_{1}<\tau<\tau_{2}$. For concave profiles, $\hat{\Theta}_{E}(\tau)$ is guaranteed to be above the linear interpolation of $\hat{\Theta}_{E}\left(\tau_{1}\right)$ and $\hat{\Theta}_{E}\left(\tau_{2}\right)$, which is a highly desirable property. On the other hand, for convex profiles, 

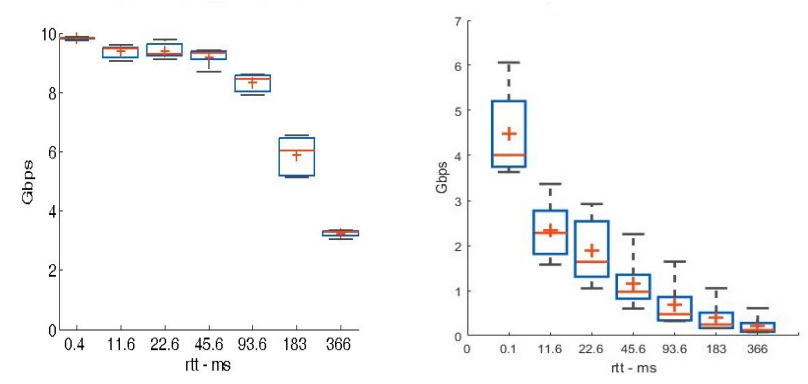

(a) XDD transfers between XFS file sys-(b) Lustre file transfers using LNet tems mounted on SSDs routers

Figure 1: Throughput profiles $\hat{\Theta}_{E}$ of file transfers over 10GigE connections

the only guarantee that can be provided is that $\Theta_{E}(\tau)$ is above the minimum of $\hat{\Theta}_{E}\left(\tau_{1}\right)$ and $\hat{\Theta}_{E}\left(\tau_{2}\right)$. At a deeper level, the shape of $\hat{\Theta}_{E}(\tau)$ reflects the time dynamics of file transfers, and in particular its concavity requires fast ramp-up followed by stable throughput [15].

Overall, the current and past measurements indicate that $\hat{\Theta}_{E}(\tau)$ is typically concave for smaller RTT and then switches to convex as RTT is increased [15-18]. Consider the memory transfer profile $\hat{\Theta}_{T}(\tau)$ of the underlying TCP transport, which itself exhibits such a dual property, typically with a wider concave region [18]. Highperformance protocols, such as Hamilton TCP [21] and Scalable TCP [8], provide wider concave regions compared to other TCP variants. Furthermore, increasing configuration parameters, such as the buffer size and number of parallel streams, leads to expanded concave regions [15], which represents a more effective transport method. The file transfer profile $\hat{\Theta}_{E}(\tau)$ is a modulated version of $\hat{\Theta}_{T}(\tau)$ by file, IO and host systems, typically resulting in smaller concave regions, as shown subsequently. In this paper, we show the dependencies of $\hat{\Theta}_{E}(\tau)$ on both network and file IO parameters, and highlight the differences among various file transfer methods, and then propose systematic methods to compare them. We mainly focus on analytics of measurements under various configurations that have described in detail in $[14-17,19]$, which we briefly summarize here in various sections to provide the context and illustrate specific performance parameters.

We summarize various file throughput measurements collected over a suite of dedicated 10 Gbps emulated connections with 0 366 ms round-trip times, using GridFTP [22] and XDD [23] file transfer tools, and Lustre file system extended over WANs using LNet routers [14]. Their collection spans a five-year period and covers a variety of combinations with large Terabyte datasets, and the individual configurations and tests have been described in detail in $[14-17,19]$. Together, these test configurations are composed of: (i) three types of host systems, 48- and 32-core data transfer servers, and 32-core cluster nodes; (ii) three file systems, host file systems ext3 on local hard disk, XFS on SSD drive, and Lustre implemented on IB network; and (iii) two types of network connections, $10 \mathrm{Gbps}$ Ethernet, and 9.6 Gbps OC192 SONET wide-area connections. As expected, in reflecting TCP transport, $\hat{\Theta}_{E}(\tau)$ decreases with RTT

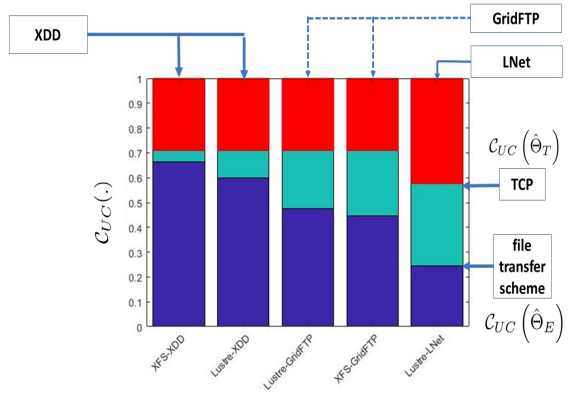

Figure 2: Summary of $C_{U C}$ for five file transfer methods

and has narrower concave regions that reflect the file transfer methods, but their comparison is complicated by different peaks and convex-concave transitions.

To provide insights into throughput profile and its trends, we present analytics based on the convexity-concavity geometry of $\hat{\Theta}_{E}(\tau)$ using the above collection of measurements as a starting point. Specifically, we propose the utilization-concavity coefficient $C_{U C} \in[0,1]$, a scalar metric that represents both peak throughput and concave region of $\hat{\Theta}_{E}(\tau)$. It is as an extension of a simpler memory transfer version in [9], and it enables an objective comparison of multiple file transfer methods. For five different file transfer methods ${ }^{1}, C_{U C}$ is illustrated in Fig. 2 in blue, wherein left- and rightmost columns represent the best and worst profiles corresponding to XDD XFS and Lustre LNet transfers in Figs. 1(a) and 1(b), respectively. The green regions represent additional throughput achieved by TCP memory transfers, and the red regions represent the connection capacity not utilized by TCP. In addition to performance comparison, our results show that these analytics lead to performance optimizations by highlighting the roles of parallelism in GridFTP and XDD, and buffer utilization and credit mechanism in LNet routers.

The organization of this paper is as follows. File systems and transfer tools are briefly described in Section 2. In Section 3, we introduce the utilization and concave-convex coefficients to characterize the file transfer throughput profiles. The testbed utilized in collecting the measurements is discussed in Section 4. Throughput measurements and profiles are presented in Section 5, and their analytics are described in Section 6. Statistical guarantee aspects of the utilization-concavity coefficient are discussed in Section 7. We conclude in Section 8.

\section{FILE SYSTEMS AND TRANSFER TOOLS}

A wide-area disk-to-disk file transfer configuration encompasses storage devices, data transfer hosts, and local- and wide-area connections, as illustrated in Fig. 3. Several sites often use dedicated data transfer hosts, such as Data Transfer Nodes (DTNs), along with the high-performance Network Interface Cards (NICs) to access network connections and the Host Channel Adapters (HCAs) to access network storage systems. Transfers also involve a range of software components, including the file system IO modules for disk access and the TCP/IP stack for network transport. The file

${ }^{1}$ We did not attempt to extensively optimize the individual methods, since our goal for this figure is to illustrate and highlight different profiles. 


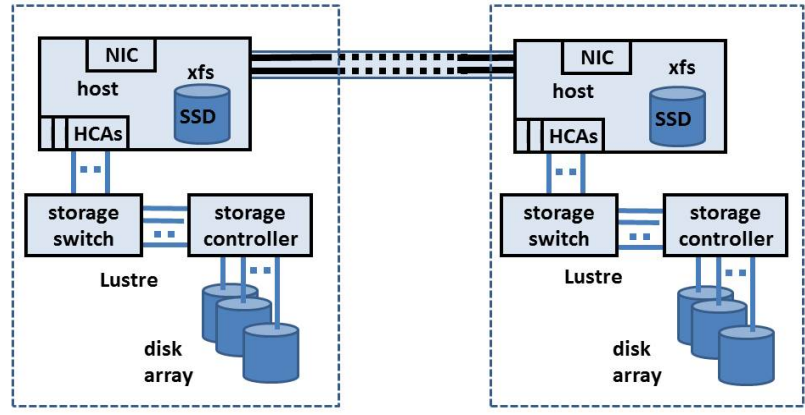

Figure 3: File transfers over long-haul connections

throughput profile $\hat{\Theta}_{E}($.$) is composed of the underlying TCP profile$ $\hat{\Theta}_{T}($.$) and the local file throughput \hat{\Theta}_{H}($.) at the host $H$. Typically, $\hat{\Theta}_{E}(\tau) \leq \hat{\Theta}_{T}(\tau)$ and they both decrease with $\tau$, and $\hat{\Theta}_{H}$ at end nodes limits the peak throughput of $\hat{\Theta}_{E}(\tau)$, which is achieved at lower RTT $\tau$. The resultant performance critically depends on the parameters of file IO and network transport, which are parts of the file transport method. In particular, parallel operations are used to increase the effective throughput of both file systems and wide-area network transport, typically by utilizing a set of buffers. We will now briefly describe the coordination of these two critical systems by XDD, GridFTP, and LNet routers.

\subsection{XDD File Transfers}

For file transfers, XDD spawns a set of threads to open a file and perform data transfers between storage and network. A source XDD process creates a TargetThread that opens the file, initiates a connection with a destination XDD process, and subsequently creates a number of QThreads that issue read commands to fill a thread-local buffer. Once a thread's buffer is filled, that thread transmits the data over the network to a destination XDD process. Similarly, the destination XDD process initiates a thread that listens for a connection from a source XDD process and then creates QThreads to receive data from the network and write the data into the storage system. The number of source and destination QThread pairs is equal to the number of TCP parallel streams. XDD reports read transfer rate at the sender and write transfer rate at the receiver for each file transfer by aggregating across all flows. This tight pairing of IO and TCP streams is particularly effective in Lustre file systems when the number of flows matches the number of stripes used for file operations [16].

\subsection{GridFTP File Transfers}

GridFTP is an extension of the standard File Transfer Protocol (FTP) for high-speed, reliable, and secure data transfer [1]. It implements extensions to FTP, which provide support for striped transfers from multiple data sources. Data may be striped or interleaved across multiple servers, as in a parallel file system such as Lustre. GridFTP supports parallel TCP flows via FTP command extensions and data channel extensions. A GridFTP implementation can use long virtual round trip times to achieve fairness when using parallelism or striping. In general, GridFTP uses striping and parallelism in tandem, i.e., multiple TCP streams may be open between each of the multiple servers participating in a striped transfer. However, this process is somewhat different compared to XDD wherein each IO stream is handled by a single TCP stream, whereas such association is less strict in GridFTP.

\subsection{Lustre over Wide-Area Networks}

Lustre file system employs multiple Object Server Targets (OSTs) to manage collections of disks, and multiple Object Storage Servers (OSSs) to stripe file contents. Lustre clients and servers connect over the network, and are configured to match the underlying network modality, for example IB or Ethernet. Host systems are connected to IB switch via HCAs, and Lustre over IB clients is used to mount the file system on them over IB connections. Due to a latency limit of $2.5 \mathrm{~ms}$, such deployments are limited to site-level access, and do not provide file access over wide-area networks. This IB-based Lustre file system is augmented with Ethernet Lustre clients, and LNet routers are utilized to make IB-based OSSs available over wide-area Ethernet connections [14]. Compared to GridFTP and XDD that are software applications, the implementation of LNet routers requires more changes to the infrastructure.

\subsection{Host and TCP Profiles}

We now consider cases that illustrate the parameters that affect host profiles $\hat{\Theta}_{H}$ and TCP profiles $\hat{\Theta}_{T}$, which in turn affect $\hat{\Theta}_{E}$.

2.4.1 Host Profiles. To establish baselines for $\hat{\Theta}_{H}$ we consider IOzone measurements collected for four host configurations:

(i) feynman:32-core workstation with ext3 file systems mounted on local hard disks,

(ii) bohr: 48-core server with XFS file system mounted on SSD drives connected over PCI bus,

(iii) bohr: 48-core server with Lustre mounted on IB and Ethernet using LNet routers, and

(iv) tait: 32-core cluster node with Lustre mounted on IB and Ethernet using LNet routers.

IOzone disk write measurements are shown in Fig. 4 corresponding to different transfer sizes and segment sizes for cases (i) and (ii). For ext3 in case (i), the throughput is a function of the transfer size as shown in Fig. 4(a), wherein throughput rates are much higher for smaller sizes but are limited to $2 \mathrm{Gbps}$ for $10 \mathrm{~GB}$ transfers that access the disk system. For XFS on SSD, the write throughput rates are about $10 \mathrm{Gbps}$, and are maintained for $10 \mathrm{~GB}$ data transfers as shown in Fig. 4(b), thereby illustrating the higher speed of SSD devices.

For Lustre under IB and Ethernet configurations, IOzone throughput measurements are shown in Fig. 5. The peak write throughput $\hat{\Theta}_{H}$ of 6 Gbps is achieved in both cases in transferring datasets of size $1 \mathrm{~GB}$ by using a sufficiently large segment size. However, $\hat{\Theta}_{H}$ is maintained around peak 6 Gbps in IB configuration and significantly decreased for LNet configuration for 2 GB (or larger) data transfers. In particular for $10 \mathrm{~GB}$ transfers, $\hat{\Theta}_{H}$ in the latter case decreases to around 2 Gbps, whereas it is maintained at 6 Gbps in IB configuration, and increasing the number of stripes from 2 to 8 has a limited effect. These LNet router effects are critical in determining not only the peak but also the convexity of $\hat{\Theta}_{E}(\tau)$ as will be discussed subsequently. Thus, IOzone measurements highlight the 


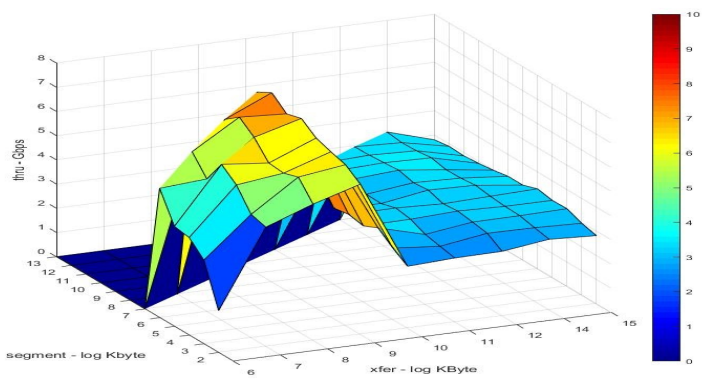

(a) ext3 mounted on local hard disk

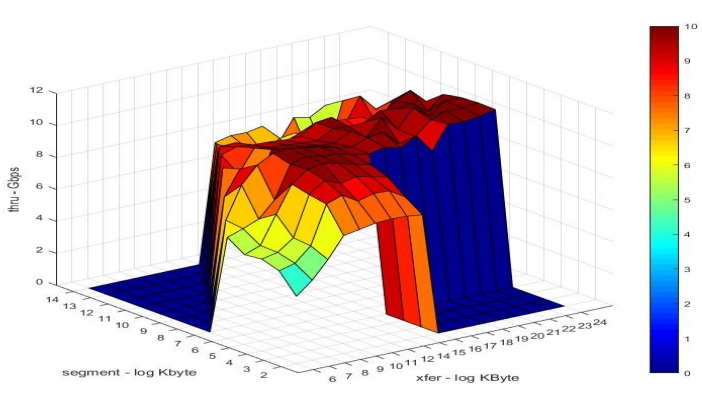

(b) xfs mounted on SSD

Figure 4: IOzone write throughput measurements: ext3/xfs

critical differences between $\hat{\Theta}_{H}$ under different file systems and storage media.

2.4.2 TCP Profiles. We obtain $\hat{\Theta}_{T}$ for two classes of hosts (a) 48-core and 32-core stand-alone systems (bohr and feynman, respectively), which are typically used for data transfers, and (b) 32-core hosts (tait) which are nodes of a compute cluster typically used for computations. The hosts are configured to use Hamilton TCP (HTCP) [21] in most of our tests, and their buffer sizes are set at largest allowable values. TCP parameters of data transfer hosts are tuned for wide-area connections, which achieve peak memory transfer rates measured by iperf shown in Fig. 6(a). On the other hand, cluster nodes have default parameters that achieve much lower memory transfer rates shown in Fig. 6(b). To support LNet connections, their TCP buffers are changed to the larger values to avoid TCP being the throughput bottleneck. The resultant shape of $\hat{\Theta}_{T}$ reveals critical performance parameters in addition to improved throughput. The improved profiles are concave for lower RTT and switch to convex for higher RTT, as illustrated in Fig. 6(a). For small RTT, the buffers are sufficiently large to maintain peak flow rates, but the limit the amount of data in transit for larger RTT, which is analytically shown to result in a convex profile [15]. Indeed, the small TCP buffers of cluster nodes prior to tuning limited in-transit data, which resulted in the very prominent convex profile in Fig. 6(b)

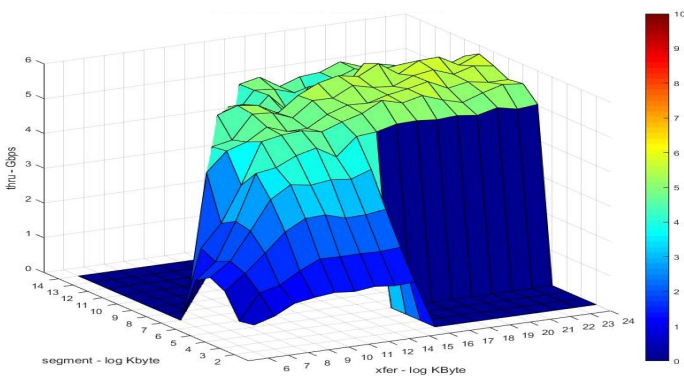

(a) Lustre IB clients supported by site IB network

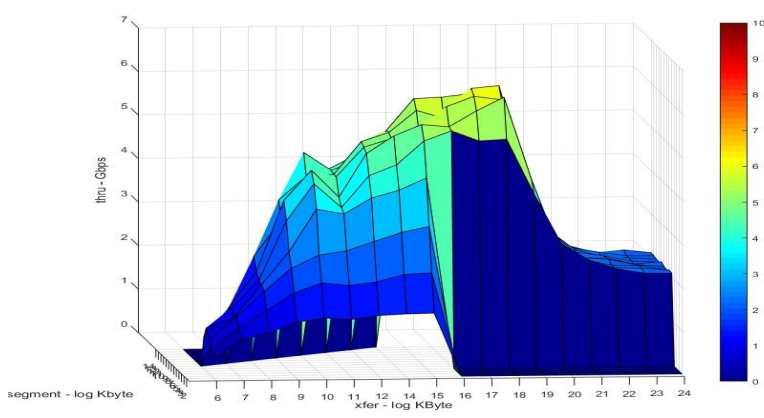

(b) Lustre Ethernet clients supported by Lnet routers

Figure 5: IOzone write throughput measurements: Lustre
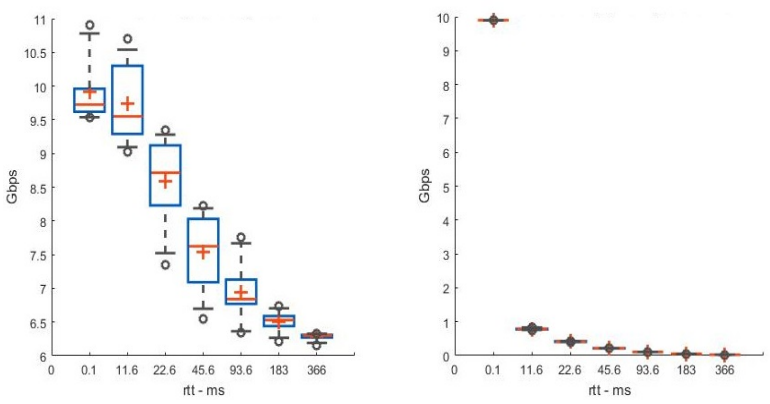

(a) 48-core data transfer hosts with tuned(b) 32-core cluster nodes with default TCP parameters TCP parameters

Figure 6: Throughput profiles of TCP memory transfers for data transfer and cluster nodes

\section{THROUGHPUT PROFILES AND COEFFICIENTS}

The end-to-end throughput profile $\hat{\Theta}_{E}($.$) is composed of the under-$ lying TCP profile $\hat{\Theta}_{T}($.$) and the local file throughput at end hosts$ $H_{i}, i=1,2$, which are quite varied and complex by themselves, as shown in the previous section. For a dedicated connection of capacity $L$ and RTT $\tau$, we have the following boundary conditions:

(i) $\hat{\Theta}_{T}() \leq$.$L , since TCP throughput is at most and typically$ lower than the connection capacity $L$; 

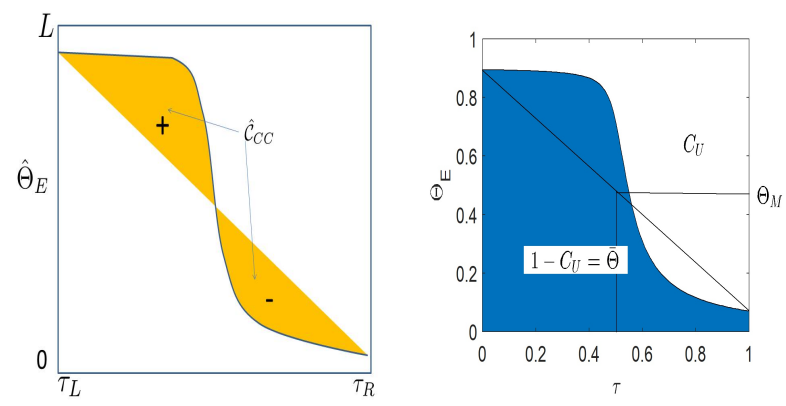

(a) convex-concave coefficient $C_{C C}$ of (b) under-utilization coefficient $C_{U}$ of $\hat{\Theta}_{E}$ $\tilde{\Theta}_{E}$

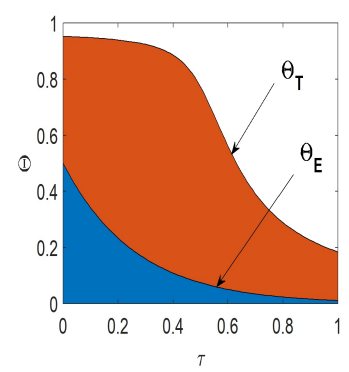

(c) network $\tilde{\Theta}_{T}$ and end-to-end $\tilde{\Theta}_{E}$

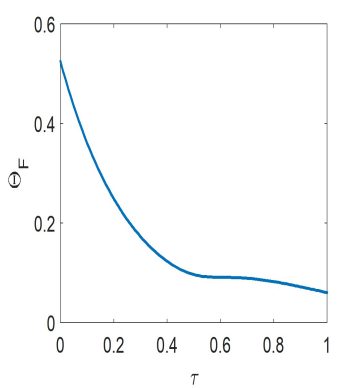

(d) file transfer $\tilde{\Theta}_{F}(\tau)$
Figure 7: Throughput profiles and coefficients

(ii) $\hat{\Theta}_{E}(.) \leq \hat{\Theta}_{T}($.$) in most cases, since TCP memory transfers$ between the hosts are not constrained by the file systems; and

(iii) $\hat{\Theta}_{E}(.) \leq \min \left\{\hat{\Theta}_{H_{1}}, \hat{\Theta}_{H_{2}}\right\}$, since the file transfer rates are limited by local file system throughput at both ends of the connection.

In what follows, we first review the key coefficients that have been first proposed in [9] for $\hat{\Theta}_{T}($.$) and extend them to end-to-end$ throughput $\hat{\Theta}_{E}($.$) . Then, we describe the profile of the file transfer$ scheme $\hat{\Theta}_{F}($.$) and its coefficients.$

\subsection{Convex-Concave and Utilization-Concavity Coefficients}

Let $\hat{\Theta}:\left[\tau_{L}, \tau_{R}\right] \mapsto[0, L]$ be a generic throughput profile and $\tilde{\Theta}$ : $[0,1] \mapsto[0,1]$ be its "normalized" version such that

$$
\begin{gathered}
\tilde{\Theta}(\tau)=\frac{1}{L} \hat{\Theta}(\tau) \\
\tilde{\Theta}(\tilde{\tau})=\frac{1}{L} \hat{\Theta}\left(\tau_{L}+\left[\tau_{R}-\tau_{L}\right] \tilde{\tau}\right),
\end{gathered}
$$

wherein the throughput values are scaled by $L$, and the operand $\tilde{\tau}$ is translated and scaled from interval $\left[\tau_{L}, \tau_{R}\right]$ to $[0,1]$. The convexconcave coefficient $C_{C C}$ is the sum of areas above and below the linear interpolation of $\hat{\Theta}\left(\tau_{L}\right)$ and $\hat{\Theta}\left(\tau_{R}\right)$ with positive and negative signs, respectively, as shown in Fig. 7(a). When applied to normalized profile $\tilde{\Theta}$, it has the following simple form [9]

$$
C_{C C}(\tilde{\Theta})=\left[\overline{\tilde{\Theta}}-\frac{\tilde{\Theta}\left(\tau_{L}\right)+\tilde{\Theta}\left(\tau_{R}\right)}{2}\right]=\tilde{\tilde{\Theta}}-\tilde{\Theta}_{M},
$$

which is the difference between the mean and mid-point $\tilde{\Theta}_{M}=$ $\frac{\tilde{\Theta}\left(\tau_{L}\right)+\tilde{\Theta}\left(\tau_{R}\right)}{2}$, and takes values in the range $\left[-\frac{1}{2}, \frac{1}{2}\right]$. The underutilization coefficient $C_{U}$ represents the unutilized capacity such that

$$
C_{U}(\tilde{\Theta})=1-\int_{0}^{1} \tilde{\Theta}(\tau) d \tau
$$

as shown in Fig. 7(b).

We combine both utilization and convex-concave properties in the utilization-concavity coefficient as

$$
C_{U C}(\cdot)=\frac{1}{2}\left(\left[1-C_{U}(\cdot)\right]+\left[\frac{1}{2}+C_{C C}(\cdot)\right]\right) .
$$

In general, it takes values in $[0,1]$, such that higher values indicate higher utilization and higher concavity level, and will be used in the following sections to compare the performance of different file transfer schemes and methods.

\subsection{File Transfer Scheme Profiles}

The normalized profile of the file transfer method $\tilde{\Theta}_{E}(\tau)$ and its underlying TCP profile $\tilde{\Theta}_{T}(\tau)$ are shown in Fig. 7(c) for a typical case. The gap between them captures the effects of file and storage end systems, and also the file transfer scheme used to support the transfers between them. Since $\tilde{\Theta}_{E}(\tau)$ encompasses TCP profile $\tilde{\Theta}_{T}(\tau)$ and host profiles $\tilde{\Theta}_{H_{i}}, i=1,2$, the effectiveness of the file transfer scheme by itself can be assessed by normalizing with respect to the other two profiles as follows. We consider that TCP profile is a non-increasing function of $\tau$, and consider two cases: (a) If file throughput at both end systems is high enough such that $\tilde{\Theta}_{H_{i}} \geq \tilde{\Theta}_{T}(\tau)$, for all $\tau \in[0,1], i=1,2$, we define the throughput profile of the file transfer scheme as $\tilde{\Theta}_{F}(\tau)=\tilde{\Theta}_{E}(\tau) / \tilde{\Theta}_{T}(\tau)$, for $\tau \in[0,1]$, as illustrated in Fig. 7(d). (b) If the file system at one of sites limits TCP throughput such that $\min \left\{\tilde{\Theta}_{H_{1}}, \tilde{\Theta}_{H_{2}}\right\}<\tilde{\Theta}_{T}(0)$, we define

$$
\tilde{\Theta}_{F}(\tau)=\frac{\tilde{\Theta}_{E}(\tau)}{\tilde{\Theta}_{T}(\tau) \min \left\{\tilde{\Theta}_{H_{1}}, \tilde{\Theta}_{H_{2}}\right\}},
$$

for $\tau \in[0,1]$. In both cases, the utilization-concavity coefficient of the file transfer scheme is given by $C_{U C}\left(\tilde{\Theta}_{F}\right)$.

\section{NETWORK TESTBED WITH FILE SYSTEMS}

Our network testbed consisting of 32-core (feynman1, feynman2, tait 1 and tai t2) and 48-core (bohr05 and bohr06) Linux workstations, QDR IB switches, and 10 Gbps hardware connection emulators. For various network connections, hosts with identical configurations are connected in pairs over a back-to-back fiber connection with negligible $0.01 \mathrm{~ms}$ RTT and a physical $10 \mathrm{GigE}$ connection with 11.6 ms RTT via Cisco and Ciena devices, as shown in Fig. 8(a). We use ANUE devices to emulate 10GigE connections with RTTs $\tau \in\{0.4,11.8,22.6,45.6,91.6,183,366\} \mathrm{ms}$. We chose these RTT values to represent three scenarios of interest: lower values represent cross-country connections, for example, between facilities across the US; 93.6 and 183 ms represent inter-continental connections, for example, between US, Europe, and Asia; and $366 \mathrm{~ms}$ represents a connection spanning the globe, which is mainly used as a limiting case.

Memory-to-memory throughput measurements for TCP are collected using iperf. Typically, 1-10 parallel streams are used for 


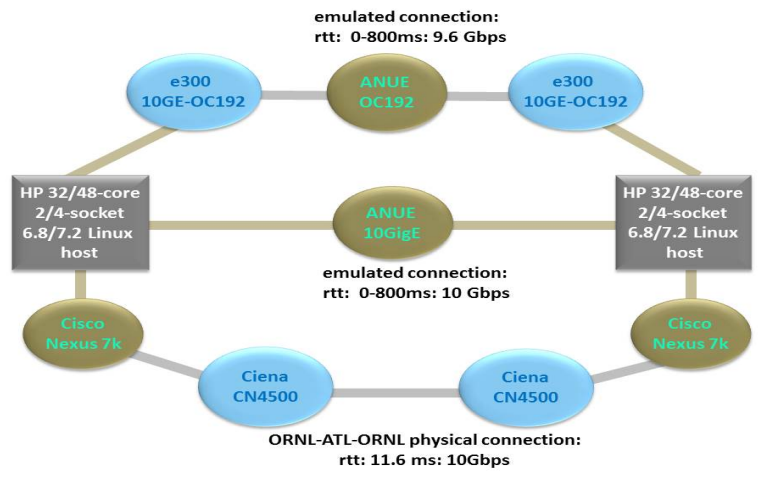

(a) Physical and emulated connections between hosts

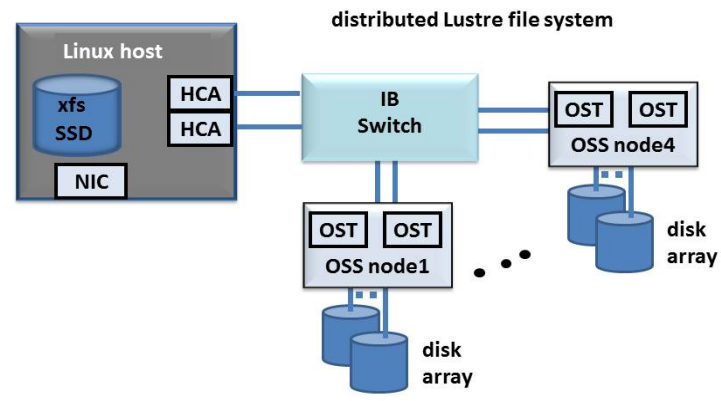

(b) Lustre and XFS file systems

Figure 8: Testbed network connections and file systems

each configuration, and throughput measurements are repeated ten times. TCP buffer sizes are set at largest allowed by the host kernel to avoid TCP-level performance bottlenecks, which for iperf is $2 \mathrm{~GB}$. These settings result in the allocation of $1 \mathrm{~GB}$ socket buffer sizes for iperf.

Our testbed consists of a distributed Lustre file system supported by eight OSTs connected over IB QDR switch, as shown in Fig. 8(b). Host systems (bohrs and taits) are connected to IB switch via HCA and to Ethernet via 10 Gbps Ethernet NICs. In addition, our SSD drives are connected over PCI buses on the hosts bohr 05 and bohr06, which mount local XFS file systems. We also consider configurations wherein Lustre is mounted over long-haul connections using LNet routers on tait 1 and bohr06, and in this case we utilize IOzone for throughput measurements for both site and remote file systems.

\section{FILE TRANSFER MEASUREMENTS}

High-performance disk-to-disk transfers between file systems at different sites require the composition of complex file IO and network subsystems, and host orchestration. For example, as mentioned earlier, the Lustre file system employs multiple OSTs to manage collections of disks, multiple OSSs to stripe file contents, and distributed MDSs to provide site-wide file naming and access. However, sustaining high file-transfer rates requires joint optimization of subsystem parameters to account for the impedance mismatches

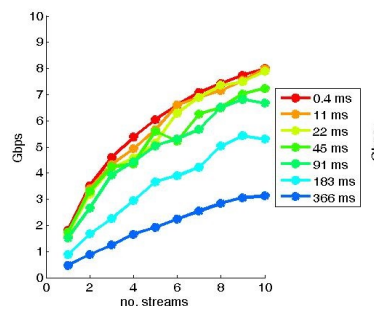

(a) 2 stripes - line plot

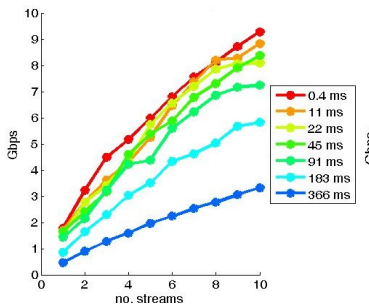

(c) 8 stripes - line plot

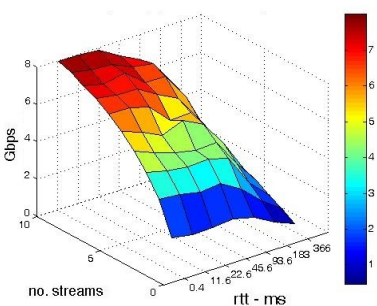

(b) 2 stripes - surface plot

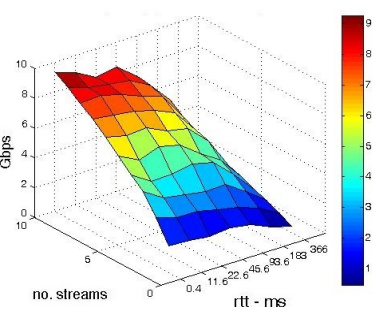

(d) 8 stripes - surface plot
Figure 9: Mean direct IO Lustre file write rates

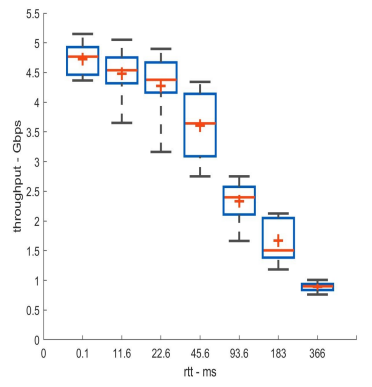

(a) 1 flow

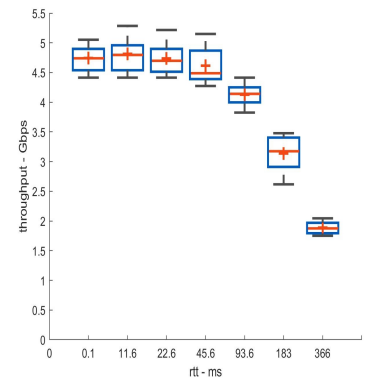

(b) 10 flows
Figure 10: GridFTP throughput for Lustre with direct IO

among them [20]. For Lustre file systems, important parameters are the stripe size and number of stripes for the files, and these are typically specified at the creation time; the number of parallel IO threads for read/write operations are specified at the transfer time. To sustain high throughput, IO buffer size and the number of parallel threads are chosen to be sufficiently large, and as we will illustrate, this simple heuristic is not always optimal. For instance, wide-area file transfers over $10 \mathrm{Gbps}$ connections between two Lustre file systems achieve transfer rates of only $1.5 \mathrm{Gbps}$, when striped across 8 storage servers, accessed with 8 MB buffers, and with 8 IO and TCP threads [16], even though peak network memory-transfer rate and local file throughput are each close to $10 \mathrm{Gbps}$.

\subsection{XDD Measurements}

We measured file IO and network throughput and file-transfer rates over Lustre and XFS file systems for a suite of seven emulated connections in the 0-366 ms RTT range (more detailed discussions on these measurements are provided in [16]). We collected two sets of XDD disk-to-disk file transfer measurements, one from XFS to 


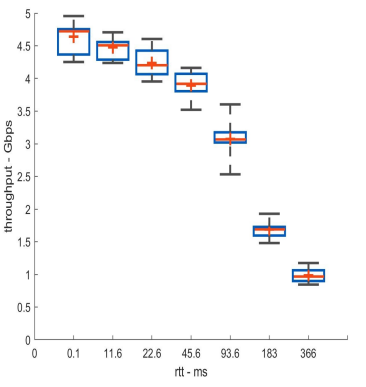

(a) 1 flow

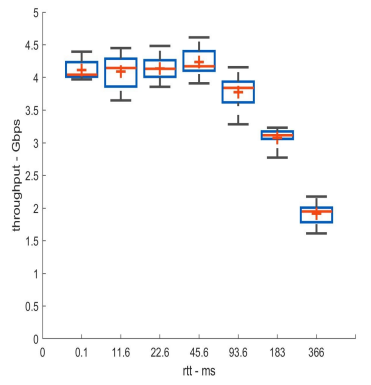

(b) 10 flows
Figure 11: GridFTP throughput for XFS over SSD drives

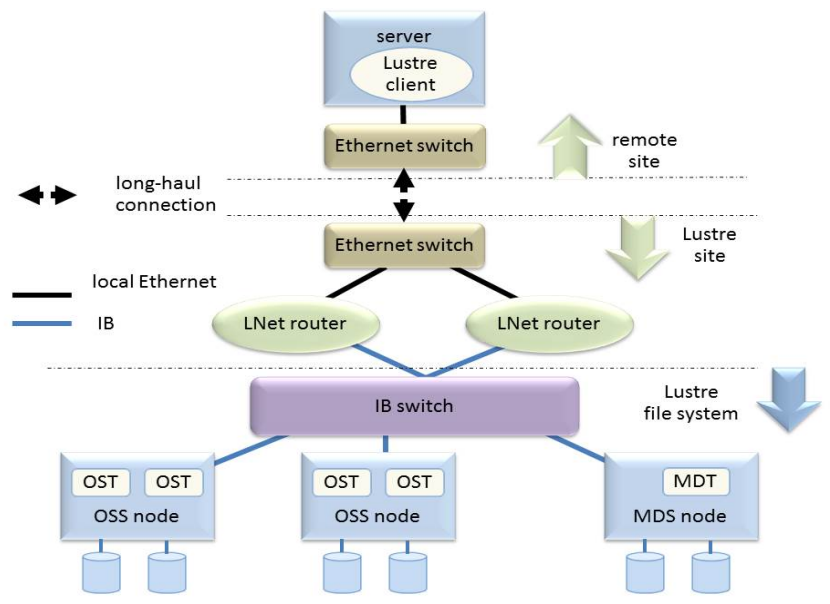

Figure 12: Lustre over long-haul connections using LNet routers between local IB and Ethernet

XFS and one from Lustre to Lustre. We considered both buffered IO (the Linux default) and direct IO options for Lustre. In the latter, XDD avoids the local copies of files on hosts by directly reading and writing into its buffers, which significantly improves the transfer rates. The mean file write throughput plots, using direct IO Lustre and either 2 or 8 stripes, are shown in Fig. 9. Results based on these and other measurements are summarized in [16]: (a) strategies of large buffers and higher parallelism do not always translate into higher transfer rates; (b) direct IO methods that avoid file buffers at the hosts provide higher wide-area transfer rates, and (c) significant statistical variations in measurements, due to complex interactions of non-linear TCP dynamics with parallel file IO streams, necessitate repeated measurements to ensure confidence in inferences based on them.

\subsection{GridFTP Measurements}

We collected measurements using GridFTP under the same configurations used for XDD measurements. Transfers of $10 \mathrm{~GB}$ files are carried out between the bohrs, where the buffer sizes are again set to be the largest allowable value and each experiment is repeated 10 times. Figs. 10 and 11 show the boxplots that illustrate GridFTP throughput performance over eight stripe direct Lustre and XFS

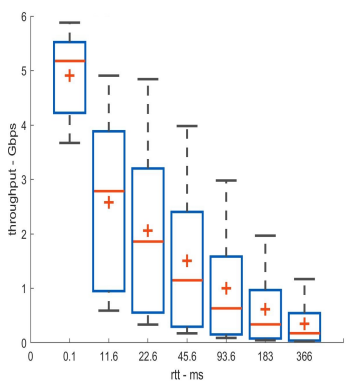

(a) 48-core stand-alone hosts

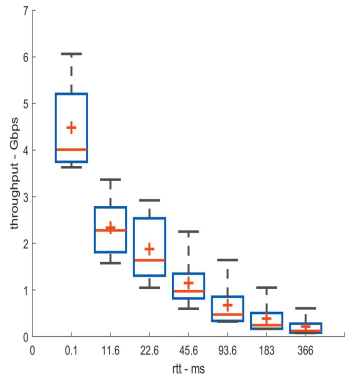

(b) 32-core cluster nodes
Figure 13: Throughput profiles of wide-area Lustre

file systems respectively, with 1 and 10 flows. From these plots, one can easily observe that the overall throughput profiles of GridFTP are somewhat lower compared to XDD (e.g., with a peak throughput below $5.5 \mathrm{Gbps}$ ); in addition, using more flows does not lead to significant increases in throughput across all RTTs, although throughputs appear more sustained with increasing RTTs under higher flow counts, resulting in somewhat more concave profile compared to the obviously convex profile under the single-flow configuration.

\subsection{Lustre with LNet Routers}

We utilize Lustre Ethernet clients on remote servers to mount file system over wide-area networks as shown in Fig. 12. At the server site, Lustre IB clients on host systems are used to connect OSS over site IB network. Some of these hosts are also connected to Ethernet Local-Area Network (LAN), which in turn is connected to WAN. These hosts are configured as LNet routers that route between IB and Ethernet. The remote hosts are connected to these hosts over the wide-area Ethernet connections. The Lustre file system is mounted at remote hosts over Ethernet such that it is functionally similar to a local Lustre system. While this configuration provides complete Lustre functionality at remote sites, file operations are performed over data paths composed of Ethernet wide-area connections and site IB connections. The data transport over wide-area connections is controlled by TCP and that over site connections is controlled by IB protocol. Consequently, the file read and write performance depends on TCP and IB parameters as well as Lustre parameters, such as LNet buffers and peer credits. Indeed these parameters must be jointly "tuned" to avoid performance bottlenecks, as will be described subsequently.

Throughput measurements using IOzone writes are shown in Fig. 13 for 10GigE emulated connections, where each measurement is repeated 10 times at each RTT value. These measurements establish that peak Lustre throughput of $1 \mathrm{Gbps}$ can indeed be achieved over connections with 366 ms RTT as shown in Fig. 13(a).

The Lustre throughput profiles are overall lower than the corresponding iperf profiles, which indicates that Lustre parameters are dominant in determining the throughput values as well as the shape of throughput profiles. The difference between the throughput of two types of hosts is within $10 \%$, indicating that it is primarily determined by Lustre parameters. More importantly, it is striking 


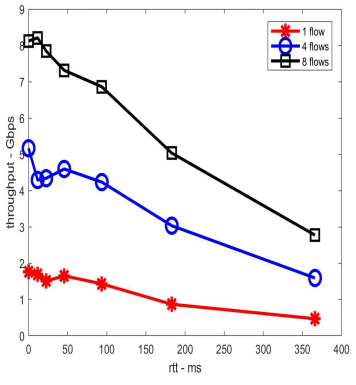

(a) throughput

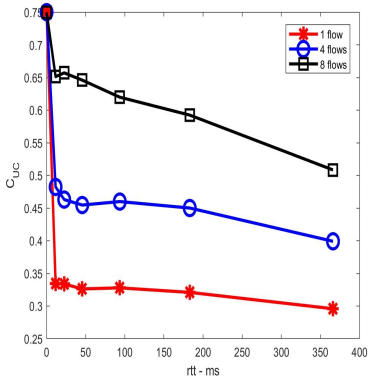

(b) $C_{U C}$

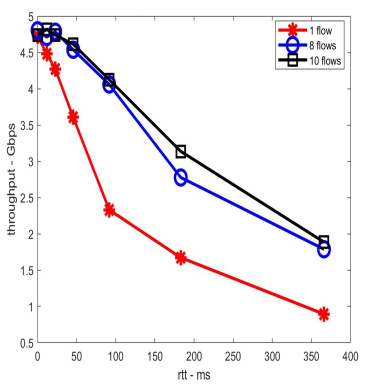

(a) throughput

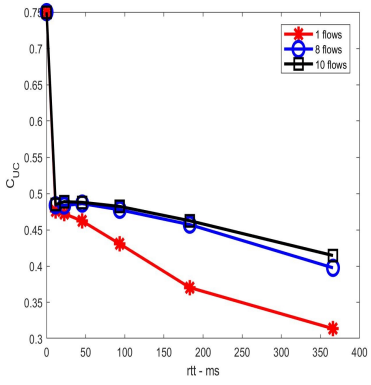

(b) $\mathcal{C}_{U C}$
Figure 14: XDD transfer analytics: direct IO Lustre, 8 stripes

that these profiles are convex, which is indicative of IO limits due to Lustre file system. Two classes of Lustre LNet parameters are critical to throughput performance: (i) LNet buffers, which limit the amount of data that can be read or written by Lustre clients, and (ii) peer credits for IB and Ethernet connections, which limit the amount of in-transit data between Lustre clients and servers. We increased buffer sizes from default $65 \mathrm{~KB}$ to $2 \mathrm{~GB}$, which only resulted in a small improvement in throughput, namely within $10 \%$. Thus, these buffer sizes are not the main contributors to the convex throughput profile. Our conjecture is that these buffers are not getting filled during the transfers due to current peer credits limit of 256. These credits must be increased separately for IB and Ethernet, which in turn requires multiple configuration changes to Lustre installation, namely, clients, LNet routers and servers. This task and testing of resultant performance will be carried out as a part of future work.

\section{FILE TRANSFER ANALYTICS}

In this section, we first analyze the end-to-end file transfer method throughput $\hat{\Theta}_{E}$ using the utilization and concavity coefficients described in Section 3. Then, we focus on the effect of the file transfer scheme throughput $\hat{\Theta}_{F}$, namely, GridFTP, XDD and LNet, by normalizing $\hat{\Theta}_{E}$ with respect to the corresponding TCP iperf measurements that constitute $\hat{\Theta}_{T}$. The normalized versions enable us to objectively compare both the utilization and concavity of various profiles, namely, network, end-to-end, and file transfer scheme throughputs, using $C_{U C}$ : its values closer to 1.0 represent high utilization and concave profile, those closer to 0 indicate lower utilization and convexity, and 0.5 represents a class of linear profiles, of which strictly linear profile is a special case.

\subsection{End-to-End Transfer Method Analytics: $\hat{\Theta}_{E}$}

Fig. 14 shows the throughput of XDD direct IO file transfers and their corresponding $C_{U C}$ curves under various RTTs and flow counts. The utilization-concavity coefficient $C_{U C}$ provides a stabilized account of the proportion of utilized link capacity, and it largely follows the trend of the throughput profile curve. For example, with 8 flows, the coefficient stays above 0.5 for all RTTs, demonstrating a much improved performance over the single-flow case, where the coefficient are only above 0.3 even for lower RTTs. Meanwhile, Fig. 15 shows the GridFTP file transfer performance
Figure 15: GridFTP transfer analytics: direct IO Lustre, 8 stripes

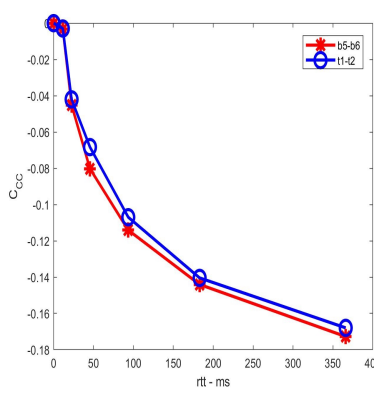

(a) $C_{C C}$

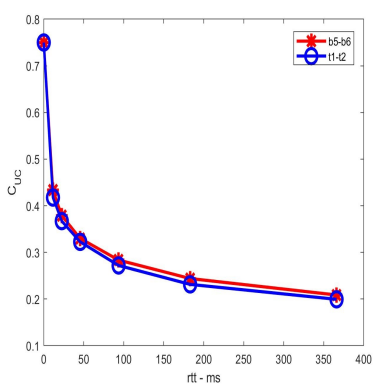

(b) $C_{U C}$
Figure 16: LNet router analytics: Lustre with 8 stripes

analytics with 8 stripe direct IO using 1, 8, and 10 flows. Compared to the previous case, here the peak throughput is below 5 Gbps and decreases with RTT, which is reflected by utilization coefficient being below 0.5 .

For LNet Lustre transfers analytics plots shown in Fig. 16, using either of (bohr or taits) pairs yields similar all-negative $C_{C C}$ curves, reflecting the convex profiles. This convexity is primarily a result of IO and Ethernet credits of LNet that only partially filled the buffers, and indeed increasing LNet buffers did not improve the performance [14]. This low utilization and convexity of LNet router transfers are indicated by $\mathcal{C}_{U C}$ values below 0.5 , reflecting the highly convex profile as seen previously in Fig. 13.

At the highest RTT, $C_{U C}$ values for XDD XFS transfers are highest among these cases, and those of LNet router are the lowest; in between these two are the XDD with direct IO Luster, GridFTP using direct IO Lustre, followed by GridFTP for XFS, as shown in Fig. 2. Thus, the utilization-concavity coefficient $C_{U C}$ is an objective indicator of the performance of file transfer methods, which takes into account the peak and concavity of throughput profiles.

\subsection{GridFTP, XDD and LNet Analytics: $\hat{\Theta}_{F}$}

The profile $\tilde{\Theta}_{F}(\tau)=\tilde{\Theta}_{E}(\tau) / \tilde{\Theta}_{T}(\tau)$ represents the effectiveness of file transfer scheme relative to TCP memory transfers, when host throughput is not a limitation. Table 1 presents the concavity coefficient $C_{C C}\left(\tilde{\Theta}_{F}\right)$ and utilization coefficient $C_{U C}\left(\tilde{\Theta}_{F}\right)$ for XFS files 
Table 1: $C_{C C}\left(\tilde{\Theta}_{F}\right)$ and $C_{U C}\left(\tilde{\Theta}_{F}\right)$ for XDD file transfer performance with XFS and 8 parallel flows

\begin{tabular}{c|ccccccc} 
RTT $(\mathrm{ms})$ & 0.4 & 11.8 & 22.6 & 45.6 & 91.6 & 183 & 366 \\
\hline point mean file transfer throughput $\hat{\Theta}_{E}(\mathrm{Gbps})$ & 9.784 & 9.532 & 9.368 & 9.191 & 8.152 & 5.636 & 2.849 \\
point mean iperf throughput $\hat{\Theta}_{T}$ & 9.905 & 9.850 & 9.793 & 9.553 & 9.208 & 8.454 & 6.660 \\
point normalized throughput $\tilde{\Theta}_{F}$ & 0.988 & 0.968 & 0.957 & 0.962 & 0.885 & 0.667 & 0.428 \\
interval mean of normalized throughput $\overline{\tilde{\Theta}}_{F}$ & 1.000 & 0.969 & 0.966 & 0.963 & 0.943 & 0.861 & 0.704 \\
interval midpoint of normalized throughput $\tilde{\Theta}_{M, F}$ & 1.000 & 0.978 & 0.972 & 0.975 & 0.937 & 0.827 & 0.708 \\
$C_{C C}\left(\tilde{\Theta}_{F}\right)$ & 0 & -0.0084 & -0.0064 & -0.0124 & 0.0061 & 0.0340 & -0.0036 \\
$C_{U C}\left(\tilde{\Theta}_{F}\right)$ & 0.7500 & 0.7304 & 0.7297 & 0.7251 & 0.7244 & 0.6976 & 0.6003 \\
\hline
\end{tabular}

Table 2: $\mathcal{C}_{U C}\left(\tilde{\Theta}_{F}\right)$ for IOzone file transfer performance with LNet Lustre and 8 stripes

\begin{tabular}{c|ccccccc} 
RTT $(\mathrm{ms})$ & 0.1 & 11.8 & 22.6 & 45.6 & 91.6 & 183 & 366 \\
\hline mean file transfer throughput $\hat{\Theta}_{E}(\mathrm{Gbps})$ & 4.908 & 2.578 & 2.063 & 1.502 & 0.998 & 0.614 & 0.349 \\
normalized end-to-end profile $\tilde{\Theta}_{E}$ & 0.491 & 0.258 & 0.206 & 0.150 & 0.100 & 0.061 & 0.035 \\
$\mathcal{C}_{U C}\left(\tilde{\Theta}_{E}\right)$ & 0.7500 & 0.4339 & 0.3792 & 0.3300 & 0.2835 & 0.2440 & 0.2087 \\
point mean iperf throughput $\hat{\Theta}_{T}$ & 9.908 & 9.735 & 8.581 & 7.535 & 6.937 & 6.510 & 6.283 \\
normalized TCP profile $\tilde{\Theta}_{T}$ & 0.991 & 0.974 & 0.858 & 0.754 & 0.694 & 0.651 & 0.628 \\
$\mathcal{C}_{U C}\left(\tilde{\Theta}_{T}\right)$ & 0.7500 & 0.7326 & 0.7333 & 0.6890 & 0.6263 & 0.5758 & 0.5332 \\
normalized file system profile $\tilde{\Theta}_{F}$ & 0.495 & 0.265 & 0.240 & 0.199 & 0.144 & 0.094 & 0.056 \\
$\mathcal{C}_{U C}\left(\tilde{\Theta}_{F}\right)$ & 0.7500 & 0.4368 & 0.3825 & 0.3440 & 0.3087 & 0.2725 & 0.2347 \\
\hline
\end{tabular}

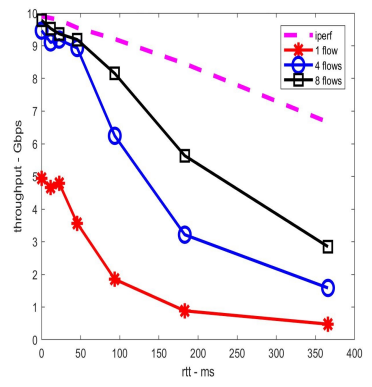

(a) throughput profiles

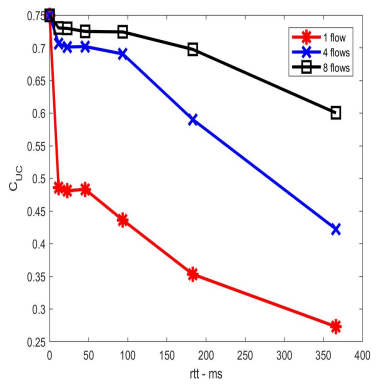

(b) $C_{U C}\left(\tilde{\Theta}_{F}\right)$

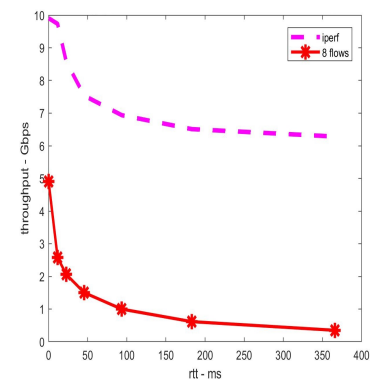

(a) throughput profiles

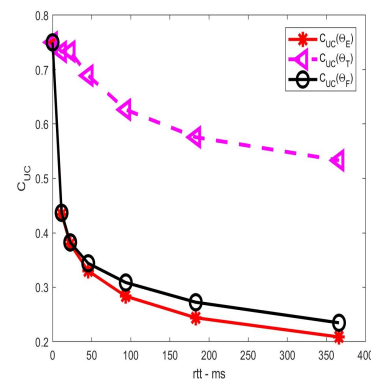

(b) $C_{U C}$
Figure 17: XDD transfer with XFS over SSD: file transfer analytics

transfers using XDD scheme; these are slightly higher compared to their end-to-end counterparts, especially at higher RTTs due to the decreasing $\tilde{\Theta}_{T}$ as shown in Fig. 17(b). The normalized throughput and $C_{U C}$ values for LNet scheme for Lustre file transfer measurements between bohrs shown in Table 2 are limited by LNet credits [14], and hence are lower than those in the above XDD cases. Compared to the nearly linear drop in throughput with increasing RTT in Fig. 17(a), in Fig. 18(a), the iperf throughput decreases more rapidly starting around intermediate RTTs, resulting in much lower $\tilde{\Theta}_{T}$ and $C_{U C}\left(\tilde{\Theta}_{T}\right)$ values; however, since the end-to-end transfer throughput values are also much lower to begin with, the resulting $\tilde{\Theta}_{F}$ is still highly convex and the improvement of $C_{U C}\left(\tilde{\Theta}_{F}\right)$ over $C_{U C}\left(\tilde{\Theta}_{E}\right)$ is far from significant as shown in Fig. 18(b).

\section{CONFIDENCE ESTIMATES}

The results in previous sections depend critically on estimates of $\mathcal{C}_{U C}(\hat{\Theta})$ in making performance inferences. These estimates are
Figure 18: IOzone measurements for LNet extended Lustre: $\tilde{\Theta}_{E}, \tilde{\Theta}_{T}$, and $\tilde{\Theta}_{F}$ analytics

subject to randomness inherent in measurements as a result of the non-linearity of transport dynamics interacting with complex host, file, and IO systems. We now derive confidence bounds for estimates of $C_{U C}(\hat{\Theta})$, which show their statistical soundness. Let $\Theta_{\tau}$ be a random variable representing the throughput of a connection in $\mathcal{S}$ with RTT $\tau \in \mathcal{S}_{\tau}$ such that $\bar{\Theta}(\tau)=\int \Theta_{\tau} d P_{\Theta_{\tau}}$ is the expected throughput. The regression performance profile $\bar{\Theta}$ is approximated by $\hat{\Theta}$ using available measurements. But, $\bar{\Theta}$ and its coefficients depend on the joint distribution $P_{\Theta_{\tau}}$, which is complex as it depends on TCP dynamics over the connection, file IO at the host, and the interactions between the two. We now show that $C_{U C}$ of $\hat{\Theta}$ is close to that of the ideal $\bar{\Theta}$ with a probability that improves with the number of measurements independent of the complex underlying distributions. The expected error in an estimate $f$ of the regression function is defined as

$$
I(f)=\int\left[f(\tau)-\Theta_{\tau}\right]^{2} d P_{\Theta_{\tau}, \tau} .
$$


We are given $l$ independently and identically distributed throughput measurements $\Theta_{\tau_{1}}, \Theta_{\tau_{2}}, \ldots, \Theta_{\tau_{l}}$. The estimator $\hat{\Theta}$ minimizes the empirical error, defined for an estimate $f$ as

$$
\hat{I}(f)=\frac{1}{l} \sum_{i=1}^{l}\left[f\left(\tau_{i}\right)-\Theta_{\tau_{i}}\right]^{2} .
$$

Then there exists a confidence function $\delta($.) such that

$$
P\{I(\hat{\Theta})-I(\bar{\Theta})>\epsilon\} \leq \delta(\epsilon, l),
$$

which shows that $\hat{\Theta}$ achieves optimal error with $\epsilon$ with confidence $\delta$ that improves with the number of measurements [15]. First, we have

$$
\left|\mathcal{C}_{U, C}(\hat{\Theta})-C_{U, C}(\bar{\Theta})\right| \leq|I(\hat{\Theta})-I(\bar{\Theta})|+\left|C_{C, C}(\hat{\Theta})-C_{C, C}(\bar{\Theta})\right| .
$$

Then, we consider the following bound

$$
P\left\{\max _{f \in \mathcal{F}}|I(f)-\hat{I}(f)|>\epsilon / 2\right\} \leq \delta(\epsilon, l),
$$

where $\mathcal{F}$ is a class of functions of bounded total variation [2], and an explicit formula for $\delta(\epsilon, l)$ can be found in Rao et al. [13]. By combining the two results, we have

$$
P\left\{\left|\mathcal{C}_{U, C}(\hat{\Theta})-C_{U, C}(\bar{\Theta})\right|>\epsilon\right\} \leq 2 \delta(\epsilon / 2, l) .
$$

which shows that the estimate $C_{U, C}(\hat{\Theta})$ of previous sections is a sound estimate of true $\mathcal{C}_{U, C}(\bar{\Theta})$. This confidence bound $2 \delta(\epsilon / 2, l)$ is distribution-free in that it is valid under all underlying distributions $P_{\Theta_{\tau}}$, and improves monotonically with the number of measurements $l$.

\section{CONCLUSIONS}

We presented a study of throughput measurements and analytics of wide-area file transfers needed by distributed science and big data computations. Extensive file throughput measurements are collected over dedicated 10 Gbps connections using GridFTP and XDD file transfer tools, and Lustre file system extended using LNet routers. The file throughput measurements were quite varied due to the complexities of host, file, IO, and disk systems, and their interactions, which make their comparison and performance optimization very challenging. We presented unifying analytics based on the convexity-concavity geometry of throughput profiles, and proposed using the utilization-concavity coefficient to characterize the overall file transfer performance. Our results provided guidelines for performance optimizations by highlighting the significant roles of buffer sizes and utilization, and parallelism implemented by file transfer methods.

Further investigations, including additional test configuration and examination of additional configurations, are needed to further improve throughput performance over shared connections. It would be of future interest to extend the calculus of throughput profiles described here in two directions, namely, deeper to focus on subsystems and broader to encompass data transfer infrastructures.

\section{Acknowledgments}

This work is funded by RAMSES project and the Applied Mathematics Program, Office of Advanced Computing Research, U.S. Department of Energy, and by Extreme Scale Systems Center, sponsored by U. S. Department of Defense, and performed at Oak Ridge National Laboratory managed by UT-Battelle, LLC for U.S. Department of Energy under Contract No. DE-AC05-00OR22725.

\section{REFERENCES}

[1] W. Allcock, J. Bresnahan, R. Kettimuthu, M. Link, C. Dumitrescu, I. Raicu, and I. Foster. The Globus striped GridFTP framework and server. In ACM/IEEE Conference on Supercomputing, pages 54-64, Washington, DC, 2005. IEEE Computer Society.

[2] M. Anthony and P. L. Bartlett. Neural Network Learning: Theoretical Foundations. Cambridge University Press, 1999.

[3] Aspera transfer service, accessed March 28, 2018. http://asperasoft.com.

[4] I. Foster and C. Kesselman. Globus: A metacomputing infrastructure toolkit. Intl 7. Supercomputer Applications, 11(2):115-128, 1997.

[5] Y. Gu and R. L. Grossman. UDT: UDP-based data transfer for high-speed wide area networks. Computer Networks, 51(7), 2007.

[6] R. Henschel, S. Simms, D. Hancock, S. Michael, T. Johnson, N. Heald, T. William, et al. Demonstrating Lustre over a $100 \mathrm{Gbps}$ wide area network of $3,500 \mathrm{~km}$. In Internationl Conference on High Performance Computing, Networking, Storage and Analysis, pages 1-8, Nov. 2012

[7] S. Jain, A. Kumar, S. Mandal, J. Ong, L. Poutievski, A. Singh, S. Venkata, J. Wanderer, J. Zhou, M. Zhu, J. Zolla, U. Hölzle, S. Stuart, and A. Vahdat. B4: Experience with a globally-deployed software defined WAN. SIGCOMM Comput. Commun. Rev., 43(4):3-14, Oct. 2013.

[8] T. Kelly. Scalable TCP: Improving performance in high speed wide area networks. Computer Communication Review, 33(2):83-91, 2003.

[9] Q. Liu and N. S. V. Rao. On concavity and utilization analytics of wide-area network transport protocols. In Proc. 20th IEEE Conference on High Performance Computing and Communications (HPCC), Exeter, U.K., Jun. 2018.

[10] Multi-core aware data transfer middleware, accessed March 28, 2018. mdtm.fnal. gov.

[11] S. Michael, L. Zhen, R. Henschel, S. Simms, E. Barton, and M. Link. A study of Lustre networking over a 100 gigabit wide area network with 50 milliseconds of latency. In 5th International Workshop on Data-Intensive Distributed Computing, page 43âĂŞ52, 2012.

[12] On-demand Secure Circuits and Advance Reservation System. http://www.es.net/oscars.

[13] N. S. V. Rao. SDN solutions for switching dedicated long-haul connections: Measurements and comparative analysis. International fournal on Advances in Networks and Services, 9(3-4), 2016.

[14] N. S. V. Rao, N. Imam, J. Hanley, and O. Sarp. Wide-area Lustre file system using LNet routers. In 12th Annual IEEE International Systems Conference, 2018.

[15] N. S. V. Rao, Q. Liu, S. Sen, J. Henley, I. T. Foster, R. Kettimuthu, D. Towsley, and G. Vardoyan. TCP throughput profiles using measurements over dedicated connections. In ACM Symposium on High-Performance Parallel and Distributed Computing, Washington, DC, July-August. 2017.

[16] N. S. V. Rao, Q. Liu, S. Sen, G. Hinkel, N. Imam, B. W. Settlemyer, I. T. Foster, et al. Experimental analysis of file transfer rates over wide-area dedicated connections. In 18th IEEE International Conference on High Performance Computing and Communications (HPCC), pages 198-205, Sydney, Australia, Dec. 2016

[17] N. S. V. Rao, Q. Liu, S. Sen, D. Towsley, G. Vardoyan, I. T. Foster, and R. Kettimuthu. Experiments and analyses of data transfers over wide-area dedicated connections. In 26th International Conference on Computer Communications and Network, 2017.

[18] N. S. V. Rao, D. Towsley, G. Vardoyan, B. W. Settlemyer, I. T. Foster, and R. Kettimuthu. Sustained wide-area TCP memory transfers over dedicated connections. In IEEE International Conference on High Performance and Smart Computing, New York, NY, Aug. 2015.

[19] S. Sen, N. S. V. Rao, Q. Liu, N. Imam, I. T. Foster, and R. Kettimuthu. On analytics of file transfer rates over dedicated wide-area connections. In First International Workshop on Workflow Science (WOWS), Auckland, New Zealand, October 2017. in conjunction with 13th IEEE International Conference on e-Science.

[20] B. W. Settlemyer, J. D. Dobson, S. W. Hodson, J. A. Kuehn, S. W. Poole, and T. M. Ruwart. A technique for moving large data sets over high-performance long distance networks. In IEEE 27th Symposium on Mass Storage Systems and Technologies, pages 1-6, May 2011.

[21] R. N. Shorten and D. J. Leith. H-TCP: TCP for high-speed and long-distance networks. In 3rd International Workshop on Protocols for Fast Long-Distance Networks, 2004

[22] GT 4.0 GridFTP. http://www.globus.org.

[23] XDD - The eXtreme dd toolset, accessed March 28, 2018. https://github.com/ bws/xdd. 